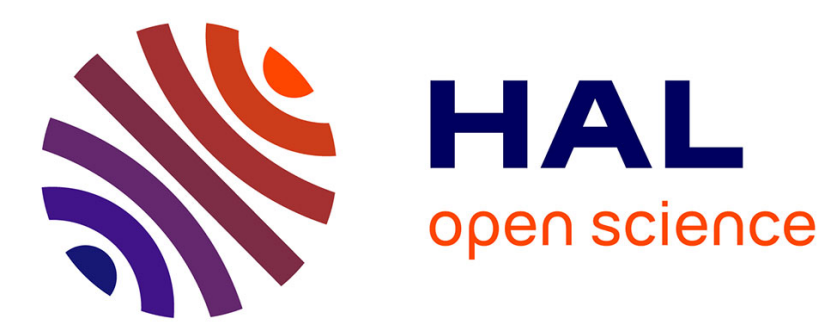

\title{
Sciences et entropocène
}

Maël Montévil

\section{To cite this version:}

Maël Montévil. Sciences et entropocène. Ecorev' - Revue critique d'écologie politique, 2021, ํ50

(1), pp.109-125. 10.3917/ecorev.050.0109 . hal-03200927

\section{HAL Id: hal-03200927 \\ https://hal.science/hal-03200927}

Submitted on 17 Apr 2021

HAL is a multi-disciplinary open access archive for the deposit and dissemination of scientific research documents, whether they are published or not. The documents may come from teaching and research institutions in France or abroad, or from public or private research centers.
L'archive ouverte pluridisciplinaire HAL, est destinée au dépôt et à la diffusion de documents scientifiques de niveau recherche, publiés ou non, émanant des établissements d'enseignement et de recherche français ou étrangers, des laboratoires publics ou privés. 


\title{
Sciences et entropocène
}

\section{Autour de Qu'appelle-t-on panser? de Bernard Stiegler}

\author{
Maël Montévil
}

En examinant le second tome de Qu'appelle-t-on panser $\left({ }^{1}\right)$, le théoricien de la biologie et épistémologue Maël Montévil, qui a collaboré avec Bernard Stiegler à la fois sur des questions théoriques et sur des expérimentations territoriales, s'arrête sur le rôle des sciences dans l'Anthropocène pour souligner leur difficulté à penser cette ère et, ce faisant, $\grave{a}$ prendre soin des vivants, humains et non-bumains, des techniques et des sciences ellesmêmes. Stiegler soulignait l'importance de la question de l'entropie, conduisant au concept d'entropocène. L'auteur introduit et illustre ce concept pour montrer sa pertinence d'un point de vue physique, biologique et social. Ce faisant, il insiste sur la parenté mais aussi sur les différences entre ces phénomènes. Dans le cas des humains, les savoirs jouent un rôle central pour lutter contre l'entropie, et les sciences pourraient retrouver leur compte en contribuant au développement-urgent - de savoirs territoriaux.

Publié comme : Montévil, Maël. 2021. "Sciences et Entropocène. Autour de Qu'appelle-t-on Panser? De Bernard Stiegler." EcoRev' 50 (1): 109-25. DOI: 10.3917/ecorev.050.0109, URL: https://montevil.org/publications/articles/2021Montevil-Stiegler-Sciences-Entropocene/

Lors de la crise sanitaire de la covid-19, qui a démarré en Chine à l'automne 2019 et se poursuit dans le monde en 2021, la contribution des sciences a été et reste marquée par une certaine confusion. Certes, les technologies d'observation ont eu un rôle presque immédiat - description initiale et séquençage du virus SRAS$\mathrm{CoV}-2$, puis tests de dépistage par PCR (Polymerase Chain Reaction) ayant une efficacité certes limitée mais maîtrisée, et enfin tests immunologiques. Pour autant, la capacité à prédire la dynamique de la pandémie - ce qui requiert un passage de la technologie à la science - a été très limitée, même pour ce qui est de la seconde vague qui a commencé en Europe à l'automne 2020. De même, l'intérêt de certains traitements possibles, des masques et du confinement n'ont pas fait l'objet, dans l'ensemble, de débats scientifiques d'une bonne qualité. Pour les vaccins qui sont au centre de l'attention depuis leur mise sur le marché à la fin de 2020, la situation semble similaire, voire aggravée par les jeux spéculatifs associés aux annonces des industriels et par les intérêts économiques colossaux associés au déploiement de tels vaccins.

Ces événements sont complexes, et ont de multiples facettes : l'activité scientifique elle-même, les interférences d'intérêts économiques dans cette activité et l'articulation avec des discours politiques, notamment gouvernementaux. Dans cet 
article, je vais me concentrer sur un de ces aspects, qui est travaillé par Bernard Stiegler dans les deux tomes de Qu'appelle-t-on panser ? : la science pense-t-elle et panse-t-elle encore?

Pour cerner cette question, retraçons rapidement son contexte. Dans le tome 2 auquel l'on se référera plus particulièrement, Bernard Stiegler examine la figure de Greta Thunberg d'un point de vue politique, en s'appuyant notamment sur le philosophe Henri Bergson pour comprendre la fulgurance de cette intervention dans la vie publique. En un mot, la position de Thunberg est double. Tout d'abord, puisque les adultes n'accomplissent plus leur tâche, les mineurs se trouvent contraints d'agir et de parler en adultes pour rappeler à ces derniers leurs responsabilités. Deuxièmement, l'essentiel du propos est simple et raisonnable : il s'agit d'écouter les scientifiques et de sortir du déni, notamment sur la question climatique.

Bernard Stiegler a été très marqué par les réactions positives mais aussi négatives à l'intervention de Thunberg dans la vie publique. Avec Jean-Marie Le Clézio, il a cherché à répondre aux appels au meurtre à son endroit - forme extrême et significative de la réaction de déni qui reste dominante dans les politiques effectives. Ceci a conduit à la création de l'Association des Amis de la génération Thunberg (AAGT). Cette association regroupe des scientifiques, et plus généralement des universitaires ainsi que des activistes venant pour l'essentiel de Youth for Climate et d'Extinction Rebellion - à ces deux groupes s'ajoutent aussi les membres d'Ars Industrialis, s'intéressant aux questions technologiques et industrielles.

Le sens de cette association est d'abord de prendre au sérieux l'injonction de Thunberg, qui rappelle l'injonction « ose savoir » d'Horace, relue par Kant et reprise ici par Stiegler. Écouter les scientifiques est une attitude raisonnable, mais la tâche est cependant plus complexe qu'il n'y paraît. S'il y a parfois des consensus scientifiques lorsqu'on considère les choses un peu grossièrement, par exemple à propos de l'existence d'un changement climatique causé par les émissions des gaz à effets de serre, le cœur de l'activité scientifique est d'abord le dissensus, qui consiste en l'espèce de juger de l'importance de tel ou tel aspect du changement planétaire, ou de la manière de comprendre et modéliser ce réchauffement. La science ne dit pas le vrai, elle n'est elle-même que lorsqu'elle accueille en son sein une pluralité de vues se confrontant au réel et se confrontant entre elles sur le plan théorique.

Sur cette base, écouter les scientifiques est une tâche qui se complexifie. Avec l'AAGT et plus généralement la recherche contributive, Bernard Stiegler ouvre une voie qui permet aux activistes, mais aussi aux professionnels et aux habitants de travailler avec les scientifiques pour produire une dynamique de réappropriation des connaissances et de développement de savoirs, à même de permettre à la société de bifurquer de sa trajectoire mortifère à tous les niveaux.

Cet agencement entre différents publics a aussi pour intérêt de donner une nouvelle perspective au travail universitaire et ainsi d'en prendre soin. En effet, ce travail est fragilisé, et Bernard Stiegler interroge fermement la capacité de la 
science contemporaine à se penser et à penser. Il y a des raisons extrinsèques à cette situation - depuis plus de quinze ans des mouvements appellent à sauver la recherche et l'Université sans recevoir de réponse politique positive, quelles que soient les majorités politiques. N'ayant pas été sauvée, l'activité scientifique est aujourd'hui significativement dégradée.

Plus profondément, l'organisation des sciences contemporaines est problématique, car elle est régie par des calculs détachés du contenu scientifique. Pour les sciences appliquées, ces paramètres concernent la contribution à l'économie, plus précisément la compétitivité sur les marchés internationaux, et, pour les sciences fondamentales, leur capacité à susciter des « citations " de collègues à court terme (deux ans), l'équivalent, dans le monde journalistique, des reprises d'une information par d'autres médias. Dans l'enseignement supérieur, la décision par le calcul consiste à fermer les filières n'ayant pas un nombre suffisant d'étudiants, ou ne plaçant pas suffisamment ces derniers sur le marché du travail. Cette (dés-)organisation conduit à éliminer des filières en faible demande alors même que des cursus dominant quantitativement en dépendent scientifiquement. Par exemple, l'ensemble de la biologie dépend de la biologie de l'évolution, ne serait-ce que parce que cette dernière classifie le vivant et donc nomme les objets de la biologie - les êtres vivants. N'ayant que peu de débouchés dans le privé, ces recherches tendent à être éliminées des offres de formation, ce qui met logiquement en péril la connaissance biologique elle-même. Dans le même ordre d'idées, mais au niveau de la recherche, le directeur de recherche Bruno Canard (CNRS), spécialiste des coronavirus, a témoigné de l'incapacité des institutions à financer des recherches sur ces virus alors que leur importance pour prendre soin de la société est aujourd'hui évidente à tout le monde. Il ne s'agit pas ici d'une critique purement rétrospective car il y a eu un certain nombre de départs épidémiques qui, s'ils ne sont pas devenus pandémiques, ont néanmoins bien montré la pertinence du sujet. La science régie par le calcul est donc en difficulté pour prendre soin de ses connaissances, de la société et du monde.

Cette difficulté est redoublée par la rapidité des changements technologiques actuels, produisant ce que Stiegler a appelé la « disruption » : une situation où la société ne parvient plus à domestiquer ses propres productions techniques dont la toxicité devient alors dominante. Dans le cas des sciences, la disruption signifie adopter tour à tour chaque nouvelle technologie en se préoccupant somme toute fort peu de la contribution de son utilisation à la connaissance et à la compréhension des phénomènes, au-delà du simple développement et déploiement de ces technologies. Le problème ne concerne pas seulement les technologies d'observation ou de calcul scientifique. Ce sont d'abord les modalités de publication, de diffusion et donc le support matériel des controverses scientifiques qui ont changé, adossés aux critères bibliométriques susmentionnés : le comptage des publications et des citations.

Or, il y a beaucoup à repenser, y compris sur le plan purement scientifique, pour comprendre et surmonter les impasses de l'Anthropocène. Bernard Stiegler insiste sur l'insuffisance de la prise en compte de l'entropie telle que décrite en 
thermodynamique, et surtout l'insuffisance de la théorisation de ses conséquences et ramifications sur les plans biologique et social.

\section{.L'entropie en physique}

Pour comprendre l'importance du concept d'entropie pour l'Anthropocène, conduisant Bernard Stiegler à appeler cette ère entropocène, une petite introduction à ce concept est nécessaire. La conception classique des sciences physiques pose un monde où rien ne se perd, rien ne se crée et tout se transforme. Par exemple, en mécanique classique, les lois pour prédire le futur et rétro-dire le passé sont exactement les mêmes, autrement dit cette théorie ne permet pas de distinguer un film lorsqu'il est passé en avant de lorsqu'il est passé en arrière. Il n'y a pas de flèche du temps et, au fond, il ne se passe pas grand-chose dans ces phénomènes. De même, il n'y a pas de théorisation des limites aux transformations que l'on peut opérer sur la matière, seulement une théorisation des moyens nécessaires pour produire telle ou telle transformation. La thermodynamique change tout cela en introduisant un nouveau cadre théorique.

Une hypothèse fondamentale de la physique est que l'énergie se conserve. Par exemple, lorsqu'on lâche une bille, elle perd de l'énergie potentielle, due à sa hauteur dans le champ de pesanteur de la terre, au profit de son énergie cinétique, due à sa vitesse. La chute libre est donc le passage de l'énergie d'une forme à une autre. Au $19^{\mathrm{e}}$ siècle, les scientifiques se penchent sur un phénomène épineux : comment comprendre ce que l'on appelle la chaleur et la température. On observe bien qu'un travail mécanique peut produire de la chaleur par friction. On observe aussi que l'on peut utiliser la chaleur pour fournir un travail mécanique, ce qui conduit notamment à la machine à vapeur. Mais on observe aussi que cette dernière opération n'est possible que lorsque la chaleur passe d'un corps chaud à un corps froid, le contraire ne se produisant jamais spontanément. On ne peut donc rien faire avec un corps à température ambiante.

La thermodynamique vise précisément à comprendre ces phénomènes, et la composante originale de cette théorie est l'introduction d'une grandeur appelée « entropie ». Il s'agit d'une grandeur qui n'est pas mesurable directement, mais qui décrit la « qualité » de l'énergie d'un système. Par exemple, lorsque les molécules constituant l'air bougent surtout dans la même direction, il y a du vent qui est utilisable par un voilier ou une éolienne. L'entropie est faible. En revanche, lorsque ces molécules vont en tous sens, il n'y a pas de vent mais il fait chaud, ces dispositifs sont inutilisables même dans le cas où l'air possède plus d'énergie que dans le cas précédent. L'entropie est élevée.

À ce stade, il est important d'énoncer les choses précisément. Le second principe de la thermodynamique stipule que l'entropie d'un système isolé ne peut qu'augmenter jusqu'à atteindre un maximum, un système isolé étant un système n'échangeant rien avec l'extérieur. Ainsi, un corps chaud en contact avec un corps froid va conduire à deux corps tièdes parce que cette dernière configuration a une entropie plus élevée. La seule manière de revenir en arrière est d'utiliser un dispositif tel qu'un réfrigérateur, demandant un apport extérieur (sous forme 
d'électricité) et donc d'ouvrir le système.

Ici, le lecteur peut se demander si l'entropie n'est pas un peu comme la vertu dormitive qui « explique », pour le médecin de Molière, l'effet somnifère de l'opium. Pour aller plus loin, décrivons un peu plus le sens de l'entropie. En un mot, l'entropie correspond à la dispersion de l'énergie au niveau microscopique. Ainsi, dans le cas où l'on a un corps chaud et un corps froid, l'énergie est concentrée dans le corps chaud. Dans le cas de deux corps tièdes, elle est dispersée dans les deux corps, et est donc plus dispersée : l'entropie est plus élevée. De la même manière, lorsqu'il y a du vent, lénergie cinétique (correspondant à la vitesse des molécules) est concentrée dans une seule direction, alors que lorsqu'il fait chaud elle est dispersée, les molécules s'agitent en tous sens. L'entropie est donc plus élevée dans le second cas. De la sorte, on comprend aussi pourquoi une balle va de moins en moins haut à chaque rebond, son énergie se disperse par friction avec l'air ; il faudrait un apport extérieur pour maintenir ce mouvement. Intuitivement, l'entropie est donc une mesure de la dispersion de l'énergie, au niveau microscopique.

La thermodynamique pose donc deux principes : la conservation de l'énergie et sa tendance à se disperser. Cette notion de dispersion peut sembler secondaire à côté d'autres processus tels que les réactions chimiques, y compris la combustion. Pourtant, elle a un caractère inexorable et, en fait, est la base de la théorie de ces phénomènes. En pratique, l'énergie étant conservée, des expressions comme " consommer de l'énergie » sont impropres ; elles proviennent sans doute d'une réduction approximative de la physique à une propriété abordable par l'économie de marché. Une voiture, certes, consomme du pétrole, mais ce faisant elle disperse l'énergie, elle ne la consomme pas. De même, un ordinateur utilise de l'énergie existante sous forme d'une différence de potentiel électrique et la disperse sous forme de chaleur tout en effectuant des calculs. Un radiateur électrique fait sensiblement la même chose, il disperse l'énergie électrique sous forme de chaleur - dans ce cas, il est important que la dispersion ne soit pas maximale et qu'elle se fasse dans une pièce si possible relativement isolée de l'extérieur. Ces exemples montrent que le discours économique posant une consommation d'énergie pour un usage (calcul, chauffer une pièce) est approximatif. Un radiateur qui ferait aussi des calculs ne demanderait pas plus de puissance électrique. Cet aspect des choses est bien plus clair lorsqu'on pose les problèmes en termes d'énergie (conservée) et de dispersion de cette énergie (augmentation d'entropie), bref en abordant les choses en s'appuyant sur notre connaissance de la nature.

Ce cadre théorique a de multiples conséquences. Il introduit l'idée que les transformations physiques ont un caractère irréversible, et ne peuvent pas être mobilisées ad libitum - notamment contre l'idée qu'un mouvement perpétuel serait possible. Elle conduit aussi à l'idée de la mort thermique de l'univers, l'idée que l'entropie de l'univers augmente, donc que son énergie se disperse, et que de moins en moins de phénomènes macroscopiques sont possibles. Mais ce qui nous intéresse ici avant tout, ce sont les conséquences de cette théorie pour le vivant, y compris le vivant humain, que Stiegler appelle « la forme noétique de la vie ». 


\section{.L'entropie et le vivant}

Nous pouvons maintenant revenir sur la rencontre manquée entre Bernard Stiegler et Aurélien Barrau relatée dans ce tome 2 de Qu'appelle-t-on panser?. Après une conférence, Barrau a critiqué l'idée selon laquelle la question de l'entropie est au cœur de l'Anthropocène, en posant qu'un des meilleurs moyens pour lutter contre l'entropie serait de bétonner l'Amazonie. Bernard Stiegler a relaté aussi que j'anticipais ce genre d'objection, en prenant comme exemple une glaciation totale de la terre comme situation baissant radicalement l'entropie et les taux d'entropie sur terre - ces deux situations étant fort peu désirables du point de vue du vivant dans son ensemble. Cette réponse était prévisible, car elle est le résultat d'une appréhension purement physico-mathématique de la question de l'entropie, en prenant la production d'entropie sur terre, par exemple, comme quantité à minimiser, effaçant ainsi la perspective du vivant. Avant d'entrer plus en détail dans l'analyse de cette question, remarquons tout de même que si le problème communément désigné comme celui de la consommation d'énergie fossile est bien une préoccupation centrale, alors il est mieux décrit en termes d'entropie - il s'agit là d'un point de vue parfaitement canonique en physique. Ici, je rejoins tout à fait Bernard Stiegler en qualifiant la réponse de Barrau comme caractérisant un refus d'obstacle : il y a certes des difficultés théoriques, mais la pertinence du concept d'entropie ne saurait être niée. Lutter contre l'entropie, chez Stiegler, ne signifie néanmoins pas minimiser la production d'entropie sur terre, et encore moins vaincre l'entropie, ceci étant impossible à cause du second principe : cette lutte ne peut être qu'une lutte tragique, à l'opposé de l'optimisation physique et des utopies calculatoires s'en inspirant en économie.

Pour aller plus loin dans l'articulation entre l'entropie et le vivant, nous pouvons partir des travaux du physicien Erwin Schrödinger $\left({ }^{2}\right)$. Un être vivant n'est pas au maximum d'entropie, mais pour se maintenir ainsi face au second principe de la thermodynamique, il dépend des flux qu'il établit avec son environnement : principalement alimentation et excrétion, parfois aussi respiration ou photosynthèse. Schrödinger suggère alors que ce qui compte dans la compréhension du vivant, c'est l'analyse de cette entropie basse et de son maintien, comment le vivant crée de "l'ordre à partir de l'ordre ».

Il faut alors passer d'une perspective purement physique à une perspective biologique, la difficulté étant qu'il n'y a pas de consensus théorique sur ces questions. Je partage le point de vue de Bernard Stiegler pour qui il ne faut pas se contenter du concept d'entropie basse capable de se maintenir grâce à des flux venant de l'extérieur, ce qui peut correspondre à des phénomènes purement physiques comme une flamme ou un ouragan. Il faut saisir comment le vivant se maintient en vie, bref son organisation. Le propre de ces organisations est qu'elles sont constituées de parties qui se maintiennent mutuellement, contre la tendance entropique, et que cette capacité est issue d'une histoire, en premier lieu l'histoire évolutive, mais aussi l'histoire d'un organisme ou d'un écosystème. Cela me permet, dans le prolongement des travaux de Baily et Longo, de définir l'antientropie, un concept proche de ce que Stiegler appelait la « néguentropie » $\left(^{3}\right)$. 
Sur cette base nous pouvons comprendre l'idée de Stiegler de lutter contre l'entropie sous toutes ses formes. En biologie, la lutte contre l'entropie correspond certes au maintien d'une entropie physique faible, mais un autre sens apparait, correspondant intuitivement à la désorganisation du vivant. Techniquement, il s'agit de la dissipation des organisations biologiques elles-mêmes, donc une entropisation de l'anti-entropie. Expliquons davantage. Les organisations biologiques sont le résultat singulier d'une histoire et leur singularité contribue à leur viabilité, à leur capacité à durer. Ainsi, des propriétés précises de l'anatomie des organes sont nécessaires à leurs fonctions. La disruption d'une organisation rend une partie de cette dernière plus aléatoire, plus générique, et ce faisant diminue la viabilité de cette organisation.

Par exemple, plantes et pollinisateurs ont des périodes d'activités saisonnières synchronisées dans un écosystème, faute de quoi les plantes ne sont pas pollinisées et les pollinisateurs n'ont pas de nourriture. Cette synchronisation est une situation singulière issue de l'histoire évolutive, elle n'aurait pas pu être le fruit d'un hasard anhistorique. Avec le changement climatique, les périodes d'activité changent, mais au lieu de changer de manière uniforme, elles se décalent de manière diverse car différentes espèces utilisent différents indices saisonniers pour démarrer leurs activités (température de l'air, du sol, durée du jour, etc.). Il s'ensuit que les périodes d'activité sont plus aléatoires, et que certaines populations sont fortement fragilisées, voire disparaissent $\left({ }^{4}\right)$. De même, mais à l'intérieur des organismes, les perturbateurs endocriniens sont des substances chimiques qui interfèrent avec l'action des hormones. Cependant, leur effet n'est pas qu'une perturbation, mais est décrit en anglais comme une disruption (endocrine disruptors). Avoir les bonnes quantités d'hormones au bon moment est essentiel pour le développement d'organes pleinement fonctionnels et les perturbateurs endocriniens viennent brouiller ces processus, conduisant à toutes sortes de problèmes allant d'un développement cérébral altéré à la difficulté à se reproduire ou au cancer. Enfin, lors des dernières décennies, l'augmentation de la transmission de maladies infectieuses d'un animal à l'autre, y compris aux humains, est un phénomène qui peut aussi s'analyser en ces termes. La transformation des interactions entre animaux par la déforestation et l'élevage intensif change profondément le milieu des agents pathogènes et de leurs hôtes.

Ajoutons qu'il ne faut pas voir les organisations biologiques comme statiques et comme le seulement le résultat d'une histoire. Elles se transforment au cours du temps en produisant de nouvelles fonctions : elles produisent de l'anti-entropie et ceci est une partie intégrante de leur capacité à durer dans le temps. Les êtres vivants se maintiennent à la fois sur la base de ce qu'ils sont à un moment donné, mais aussi en modifiant ce qu'ils sont. La sélection naturelle, mais aussi d'autres processus, tels que la plasticité développementale, permettent au vivant de faire émerger de nouvelles organisations singulières et viables. Il y a donc une course de vitesse entre les disruptions d'origine humaine et la capacité du vivant à se réorganiser, à produire de l'anti-entropie. Notons aussi que les activités humaines peuvent inhiber cette capacité du vivant à produire des nouveautés fonctionnelles et donc à se maintenir en se transformant. Par exemple, la dislocation des habitats 
empêche la circulation des populations entre les îlots restants de populations. Alors, tout se passe comme si la sélection naturelle opérait indépendamment sur de toutes petites populations. Dans ce cas, il n'y a plus suffisamment de diversité pour que ce processus permette l'adaptation. Cette disruption de l'adaptation par sélection naturelle est d'autant plus problématique que les êtres vivants doivent répondre aux autres changements de leurs milieux produits par les activités humaines.

Développons les conséquences de cette perspective. Tout d'abord, la lutte contre l'entropie au sens de Stiegler a deux composantes en biologie : 1) maintenir une entropie physique basse au sein des organisations biologiques ; 2) lutter contre la désorganisation des organisations biologiques elles-mêmes, l'entropisation de l'anti-entropie. Ces deux concepts sont liés, notamment parce que la perte de viabilité due à la désorganisation peut conduire à la mort et donc à une augmentation massive d'entropie au sens physique du terme, mais ils sont néanmoins incommensurables, car la désorganisation peut, par exemple, empêcher la reproduction, ce qui ne se réduit pas à une augmentation d'entropie physique.

Cette démarche conduit à renouveler le regard concernant l'effet des activités humaines sur le vivant. Une partie de ces effets passe par des quantités dites extensives, par exemple les êtres humains ne laissent plus assez de place aux autres espèces à cause de l'utilisation des terres par l'agriculture, les villes ou les infrastructures. Mais un autre type d'effet est d'ordre plus qualitatif, les changements d'origine humaine disrompent les organisations biologiques, $y$ compris le développement et la physiologie humaine comme dans le cas des perturbateurs endocriniens. De plus, l'analyse de ces phénomènes dépend des organisations considérées, par exemple les espèces invasives accroissent leur population et donc leur diversité, mais au prix d'une déstabilisation des écosystèmes et d'une perte de la diversité de ces derniers. L'analyse dépend donc de l'organisation décrite.

En un mot, pour comprendre la capacité du vivant à durer, il faut prendre en compte que l'histoire a conduit à des configurations spécifiques car ce qui est viable est rare parmi ce qui est possible à chaque étape de l'histoire biologique. Le vivant actuel n'est pas viable par auto-organisation spontanée, il l'est par et dans un processus historique. Réciproquement, cette historicité du vivant lui confère des vulnérabilités particulières lorsque des changements du milieu viennent éloigner les organisations biologiques de ces petites zones de viabilité par l'introduction d'aléatoire dans ces organisations. Ceci s'applique tant pour les changements chimiques du milieu, avec par exemple les perturbateurs endocriniens, que pour les changements des propriétés physiques saisonnières, dus au changement climatique. L'entropisation des organisations biologiques est donc un phénomène majeur de l'Anthropocène, qui est aussi alors un entropocène du point de vue de la biologie.

\section{.Entropie et organisations sociales}

Pour les organisations humaines, la situation a une certaine similarité avec la 
biologie. Non seulement nous devons maintenir nos processus biologiques, comme tout être vivant, mais cette activité de maintien est élargie aux objets techniques que nous produisons et dont nous dépendons. Ceci s'applique aussi bien à un couteau de cuisine qu'il faut affûter, puis remplacer, qu'aux grandes infrastructures telles que les habitations, les usines ou les réseaux électriques. Bernard Stiegler aborde cette question à la fois en continuité et en rupture avec le reste du vivant. Pour ce faire, il s'appuie sur le concept d'« exosomatisation » introduit par Alfred Lotka $\left.{ }^{5}\right)$. Lotka soutient qu'une rupture introduite par les êtres humains, par rapport à l'évolution du reste du vivant, se manifeste par la production et l'utilisation massives d'" organes » en dehors du corps tels que les couteaux et infrastructures susmentionnées.

La relation entre les êtres humains et ces objets n'est pas le résultat d'une histoire biologique, elle n'est pas entièrement déterminée par les propriétés proprement biologiques des humains. Pour leurs productions, leurs usages, leurs maintiens, leurs transformations, ces objets dépendent des savoirs humains - savoir étant entendu comme savoir-faire, savoir vivre et savoirs théoriques. Ces savoirs permettent notamment de limiter la toxicité des objets techniques, que cela soit en les transformant ou en prescrivant leur usage. Par exemple, savoir utiliser un couteau de cuisine permet de ne pas se blesser, et savoir utiliser l'informatique en science implique, par exemple, de ne pas utiliser n'importe quel calcul statistique effectué par un logiciel sans en évaluer la pertinence scientifique, et donc les limites.

Le développement de nouveaux objets techniques est toujours une déstabilisation à laquelle doivent répondre de nouveaux savoirs. La capacité à produire ces savoirs constitue, chez Stiegler, la capacité à penser qui est aussi une capacité à panser. Dans le vocabulaire introduit pour le vivant, il s'agit de la contrepartie proprement humaine de la production d'anti-entropie. La difficulté actuelle est l'accumulation de désorganisations issues des technologies tant récentes, comme le smartphone et les réseaux sociaux, que plus anciennes, comme le moteur thermique à l'origine du changement climatique, et l'incapacité à produire des savoirs pour répondre à ces toxicités. Les raisons en sont nombreuses, d'autant que ce manque de savoir touche toutes les activités humaines.

Parmi ces raisons, notons que la capacité à penser dépend elle-même du milieu technique. Ainsi, le rôle des réseaux sociaux dans l'émergence de phénomènes politiques tels que l'élection de Trump, Bolsonaro ou Modi est bien établie, et ces options politiques sont caractérisées par leur absence d'attention aux vivants tant humains que non humains. Mais de la même manière, le passage au numérique dans la publication scientifique et le management scientifique n'a pas fait l'objet d'une élaboration suffisante pour prendre soin de leurs objets, comme l'illustre l'utilisation de critères bibliométriques dans l'organisation de la recherche et de l'enseignement supérieur, évoquée en introduction. L'utilisation automatique de ces critères ne permet ni le maintien des connaissances constituées par les différentes disciplines, ni la capacité à les dépasser, notamment pour faire face à l'Anthropocène. 
Face à cette situation, la réponse défendue par Bernard Stiegler est la mise en place d'une organisation économique particulière, l'économie contributive $\left(^{6}\right)$. L'idée centrale est d'investir dans la production de savoirs, non seulement par des universitaires mais surtout par des habitants et des professionnels travaillant avec des universitaires. Par exemple, nous avons mis en place un atelier sur les écrans et la petite enfance dans le centre de Protection maternelle et infantile (PMI) PierreSemard de Saint-Denis. Le problème central abordé est la disruption des relations parents-enfants par l'utilisation des écrans, notamment les smartphones, et ses conséquences néfastes pour le développement, telles que des retards de langage, des problèmes psychomoteurs et des symptômes ressemblant à l'autisme dans les cas extrêmes. Ce travail regroupe parents, professionnels et universitaires et s'appuie sur les expériences et connaissances des différents membres du groupe pour appréhender ce problème inédit de manière autant très pratique que très théorique.

Résumons la perspective concernant l'entropie. La théorie de l'entropie en physique implique la nécessité d'étudier comment le vivant maintient une entropie basse. Cette capacité provient du maintien mutuel entre les parties d'un organisme ou d'un écosystème : leur organisation. Les organisations biologiques sont le résultat singulier de l'histoire naturelle, notamment l'évolution, et leur capacité à se maintenir dépend de cette spécificité. À l'opposé, certaines activités humaines tendent à rendre ces organisations plus aléatoires, plus génériques, bref à les entropiser, réduisant la viabilité du vivant, des écosystèmes aux corps humains. Ces changements peuvent même limiter la capacité du vivant à se réorganiser, par exemple par la disruption du processus d'adaptation par sélection naturelle. Un aspect clé de cette analyse est que les organisations viables sont rares parmi les configurations explorables par le vivant, la viabilité de ces organisations est le fruit du long processus d'évolution, et donc leur altération aléatoire conduit généralement à une perte de viabilité.

Pour les sociétés humaines, la situation est assez similaire, mais les changements techniques et les savoirs associés ont une place prépondérante. Face aux désorganisations introduites par les techniques existantes, désorganisations touchant à la fois les sociétés humaines et le reste du vivant, il n'est pas suffisant d'agir sur quelques variables à grande échelle, comme les émissions de gaz à effet de serre ou la protection de quelques espèces menacées. Comme pour la production d'anti-entropie en biologie, une activité particulière, un travail, est nécessaire pour produire les savoirs permettant aux sociétés de durer et de transformer les techniques à cette fin. Un investissement massif dans les savoirs est donc nécessaire pour prendre soin des sociétés humaines et plus généralement du vivant. Dans cette perspective, les sciences sont elles aussi l'objet de désorganisations majeures, mettant en cause leur nature même. Néanmoins, elles peuvent et doivent jouer un rôle décisif dans la production de nouveaux savoirs face aux défis inédits de l'époque. Mais pour ce faire, elles doivent renouer avec les publics concernés, en reconnaissant l'importance de leurs expériences, et s'émanciper de la logique calculatoire qui les stérilise. 
Remerciements: Une partie de ce travail est financée par la Cogito Foundation. Je remercie Victor Chaix pour la relecture d'une version précédente de cet article.

\section{.Références}

1. Bernard Stiegler. Qu'appelle-t-on panser?, 2, La leçon de Greta Thunberg, Les Liens qui Libèrent, 2020.

2. Erwin Schrödinger, Quiest-ce que la vie ?, Seuil, 1993 [1947].

3. Maël Montévil, «Entropies and the anthropocene crisis ", AI and Society, à paraittre. https://montevil.org/publications/articles/submitted-MontevilEntropies-Anthropocene/

4. Maël Montévil, «Disruption of biological processes in the anthropocene : The case of phenological mismatch », soumis.

https://montevil.org/publications/articles/submitted-Montevil-DisruptionPhenology/

5. Alfred J. Lotka, "The law of evolution as a maximal principle », Human Biology, XVII, 3, 1945, p. 167-194.

6. Bernard Stiegler (dir.), Bifurquer : il n'y a pas d'alternative, Les Liens qui Libèrent, 2020. 